\title{
INCIDENCIA DE LA PUBLICIDAD EN BUSES DE TRANSPORTE PÚBLICO DEL CANTÓN RUMIÑAHUI Y SU INFLUENCIA EN EL COMPORTAMIENTO DEL CONSUMIDOR
}

\section{INCIDENCE OF ADVERTISING ON PUBLIC TRANSPORT BUSES OF THE RUMIÑAHUI CANTON AND ITS INFLUENCE ON CONSUMER BEHAVIOR}

\author{
Rosario del C. Pineda López', Galo R. Moreno Bastidas², Roberto C Pineda L³
}

\begin{abstract}
Resumen
El objetivo de la presente investigación es determinar si la publicidad visualizada en medios de transporte, en el Cantón Rumiñahui incide favorablemente en el comportamiento del consumidor desde el punto de vista del marketing. El enfoque del estudio es mixto, el tipo de investigación es correlacional, el diseño es de campo no experimental, transversal y permite medir la correspondencia entre las variables: publicidad exterior móvil en el transporte público y las variables del comportamiento del consumidor. Se realizó una investigación de campo mediante entrevista estructurada, la cual fue validada mediante 8 expertos. Se consultó a directivos de cooperativas de buses de transporte público y se realizó una entrevista con una muestra de 383 usuarios del transporte público, mediante muestreo aleatorio de la población total de Rumiñahui. A efectos del análisis de los datos recabados, se realizó en primer lugar un análisis univariado por cada pregunta y luego bivariado con la prueba chi cuadrado para analizar la influencia entre las dimensiones de las variables. El resultado obtuvo el rechazo de la hipótesis nula planteada originalmente, concluyéndose que la publicidad en buses de transporte público influye favorablemente en el comportamiento de los usuarios del transporte público como estrategia para incentivar el consumo.
\end{abstract}

\section{Palabras claves}

Publicidad exterior móvil, buses, transporte público, comportamiento del consumidor.

Clasificación JEL: M21, M37

\begin{abstract}
The objective of the present investigation is to determine if the advertising displayed on means of transport in the Rumiñahui Canton has a favorable impact on consumer behavior from the point of view of marketing. The focus of the study is mixed, the type of research is correlational, the design is non-experimental, cross-sectional and allows measuring the correspondence between the variables: mobile outdoor advertising in public transport and consumer behavior variables. A field investigation was conducted through a structured interview, which was validated by 8 experts. Directors of public transport bus cooperatives were consulted and an interview was conducted with a sample of 383 public transport users, by random sampling of the total population of Rumiñahui. For the purpose of analyzing the data collected, a univariate analysis was performed first for each question and then bivariate with the chi-square test to analyze the influence between the dimensions of the variables. The result obtained was the rejection of the null hypothesis originally proposed, concluding that publicity in public transport buses favorably influences the behavior of public transport users as a strategy to encourage consumption.
\end{abstract}

Keywords

Mobile outdoor advertising, buses, public transport, consumer behavior.

JEL Classification: M21, M37 


\section{Introducción}

La sociedad de consumo se rige por la generación continua de necesidades, para ello, las empresas que necesitan ofertar sus productos y utilizan la publicidad como un medio muy valioso para poder lograr su principal objetivo (Flores, 2013). La publicidad se considera una estrategia útil para dar a conocer los productos y estimular su consumo. En este sentido es importante considerar que "la publicidad se puede dar en muchos medios como la televisión donde resulta un costo realmente elevado para las empresas así mismo como en la radio, estos dos medios son uno de los más costosos" (Martin y María, 2007).

En oportunidades, la reacción de los consumidores ha sido favorable y desfavorable, por esta razón, este estudio pretende determinar el comportamiento de los consumidores, en este caso del transporte público, asumiendo que puede ser un medio favorable para la publicidad. La investigación se desarrolla con un enfoque metodológico mixto, donde el medio de recolección de la opinión y percepción de los usuarios del transporte público se realizará a través de encuestas validadas por expertos en el ámbito de publicidad y transporte público.

\section{Variables de análisis}

\section{Publicidad}

Thompson (2017) afirmó que la publicidad es una manera de comunicar impersonalemente, tiene un alcance muy alto tanto en dimensión como en capacidad, esta es costeada por una empresa o patrocinador reconocido con el objetivo de informar, llamar la atención o persuadir a un grupo determinado acerca de los servicios o productos que su empresa posee.

La publicidad es una estrategia masiva y de acuerdo a los servicios requeridos por la empresa que la asume llegará a la gente que lo necesite. Estas empresas también son conocidas con el nombre de patrocinador, quienes promocionan la campaña publicitaria, para que sea efectiva de la manera en que ellos la consideren necesario.

La publicidad se da de una manera inconsciente y automática, se relaciona con términos como venta o marketing. La realidad es que los anuncios con los que se comparte la cotidianidad tienen el fin de dar a conocer los productos o servicios para su consumo o contratación, en tal sentido, la publicidad se concibe como una herramienta en diferentes presentaciones para la comunicación persuasiva (Rodríguez, 2016).

Actualmente, las personas reconocen a la publicidad con el nombre de marketing, y tiene el objetivo de dar a conocer tanto a la empresa productora como a sus servicios o productos, sean nuevos o no, que requieran dar a conocer presentado en diferentes formatos. Con la nueva era digital, la publicidad tradicional está quedando rezagada y sus estrategias se enfocan en el uso de medios tecnológicos.

Cabrejos (2017) sostiene que la publicidad es una comunicación impersonal asumida y costeada por una empresa llamada también patrocinador, que puede identificarse claramente, utiliza medios de comuniación masivos para dar a conocer su información y busca la manera de persuadir al publico objetivo o audiencia.

La empresa usa los medios de comunicación masiva para tratar de cambiar la mente del consumidor y convencerlo del producto que está promocionando o dando a conocer; en esta comunicación se transmite la información necesaria para que el consumidor conozca todo lo necesario y se interese en el producto.

\section{Comportamiento del consumidor}

Villarejo (2018) señala que los precios que están basados en una promoción sirven para 
que los consumidores se sientan atraídos y así poder orientar sus compras. Los sistemas de promociones y descuentos se basan en identificar qué factores son determinantes para el comportamiento de compra de los consumidores y así evaluar la decisión.

Los consumidores están siempre con una mentalidad abierta y dispuesta a las promociones. Al observarlas, inmediatamente analizan, consideran sus necesidades, evalúan la conveniencia y deciden la compra del producto. En este orden de ideas, el descuento hace que el consumidor cambie su mentalidad y se vea persuadido para comprar el producto.

Callejón (2016) plantea que el comportamiento del consumidor se convierte en una realidad muy evidente con el pasar del tiempo. Los canales de distribución innovan constantemente en sus propuestas para generar un ambiente de multisensaciones, estas características se ven reflejadas en el producto y en el ambiente creado en el punto o espacio de venta asociado a la promoción.

Este autor, en su propuesta, considera a los canales de distribución, los cuales gestionan la materia relacionada con los precios, la manera en que se presenta el producto hacia el cliente, hacerlo llamativo, que impregne la mente del consumidor y así estimular el interés en el consumidor.

Inannini (2018) mencionó que el marketing olfativo busca sus bases -en la actualidad- de la ciencia ya que usa olores y aromas con el único fin de afectar el comportamiento de un cliente al momento de buscar algun servicio o producto para incrementar el beneficio que puede traer para la empresa externa, como internamente. En cuanto a lo externo, para generar beneficios económicos y a lo interno para mejorar el ambiente laboral generando mas productividad y que los empleados aumenten su rendimiento.

Para llegar a la mente del consumidor e incidir en su intención de compra o consu- mo, este puede ser persuadido a través de los sentidos como son el tacto, gusto, oído o vista, esta nueva tendencia está surgiendo y se la conoce como marketing olfativo. Esta última tendencia está demostrada y fundamentada en evidencia científica. En consecuencia, las empresas no solo usan este novedoso recurso para incidir en el comportamiento del consumidor, sino para lograr que sus trabajadores sean más eficientes y productivos.

\section{Teorías de soporte}

\section{Teoría de la aguja hipodérmica}

Autores como Bauer y Bauer (1960, citados por Gálvez [2012]), mencionan que al inicio del siglo XX hubo opiniones generalizadas acerca de cómo los medios pueden llegar a moldear e influir en las actitudes que las personas tienen en cuanto a su entorno, específicamente, algo que visualizan ya sea en calles o algún lugar en específico de la ciudad. Este aspecto se debió a la popularidad adquirida por diferentes medios en ese entonces.

Esta teoría fue formulada durante los periodos de tiempo circundantes a la Primera y Segunda guerra mundiales ya que fueron momentos difíciles para los pueblos y se cree que en este tiempo las propagandas causaron efectos en las personas. Es de allí que se concluye que "los mensajes de los medios atacan y se insertan (cual aguja) en la audiencia, quiénes reaccionan de manera uniforme a dichos estímulos" (Cantoral, 2015).

Es notoria la antigüedad de la teoría y sobre todo el efecto que causó en aquellas épocas que, pese a que no se tenían muchos estudios, se llegó a conclusiones bastante lógicas y sobre todo influyentes.

Los medios de comunicación, en diferentes ocasiones, difunden información de distintos tipos y dirigidas a cierto público, se puede decir incluso que podrían tratar de 
manipular a su audiencia mediante alguna propaganda. Pero la realidad es otra, porque cada individuo discernirá la información que ha recibido de una manera totalmente distinta; por esto no llega a ser cierto el hecho de que los medios tengan la capacidad para influir de manera significativa en las personas que los sintonizan. (Cantoral, 2015).

La manera de comunicar información ha ido cambiando a través del tiempo, se ha adaptado a la época y a la tecnología existente para difundir un mensaje, pero algo muy importante también es que las personas tienen diferentes formas de asimilar lo que les llega a través de distintos medios y pese al paso del tiempo, eso es algo que se ha mantenido.

\section{Teoría sobre los efectos limitados}

Por los años treinta del siglo XX, la teoría de la aguja hipodérmica es cuestionada. Se reconoce la influencia de los medios de forma individual e inmediatamente se efectúan estudios, lo cuales se actualizan e innovan con el transcurso de los años, considerando novedosos metodologías y estudios sobre la influencia de nuevos tipos de variables. (Gálvez, 2012)

El paso del tiempo ha permitido realizar estudios que concedan fiabilidad a teorías como la de los efectos limitados, permitiendo que quienes la aplican puedan innovar y adaptarla. Los medios tienen influencia significativa en el entorno y mediante esta teoría se ha comprobado.

En este sentido, Lazarsfeld, con respecto a esta teoría, realizó estudios con personas al azar, evidenciando que existe un grupo de personas que tienen una influencia significativa en los demás y son más proclives a que la gente los siga. La causa más probable a esto es que tienen un alto conocimiento sobre el tema tratado. (Asenjo, 2019).

El hecho de que una persona influya en una tercera no es algo nuevo, varios experimentos y teorías explican cómo una persona llega a verse influenciada por alguien de su entorno o simplemente alguien famoso en el momento que va a tomar una decisión. En consecuencia, es importante considerar que:

Cada individuo interpreta los mensajes de los medios, pero a diferencia de la teoría de la aguja hipodérmica, estos mensajes pasan por un filtro personal según las características psicológicas, el contexto social del individuo y sus hábitos de percepción (Teoriascom, 2015).

Los mensajes difundidos por distintos medios llegan a gran variedad de personas con diferentes personalidades, gustos y formas de pensar por lo tanto su forma de asimilarlo será diferente y hay que aprender a diferenciarlos.

\section{Teorías del comportamiento del consumidor}

\section{Teoría psicológica social}

Autores como Arévalo, Garizabal y Campo (2001, citados por Bardales [2017]), manifiestan que esta teoría tiene un enfoque psicosociológico. Esto se explica que claramente en las personas influyen, mucho más que solo el aspecto económico, las necesidades, deseos y otras particularidades y características psicológicas individuales. Por otro lado, terceras personas también afectan al comportamiento tales como miembros del grupo familiar, amigos o compañeros.

Muchas teorías abarcan el tema psicológico de las personas al momento de asumir decisiones. Sin embargo, esta se centra más al hecho de que no solo lo psicológico influye, sino también lo social, haciendo referencia a la intervención de terceras personas que inciden en el proceso de decidir sobre aspectos relevantes. En tal sentido,

Este modelo considera al hombre como un animal social adaptado a las normas de su cultura, y que sus deseos y con- 
ducta están forjados por afiliaciones a los grupos actuales o por deseo de pertenecer a aquellos de quiere alcanzar. (Consumer Behavior, 2014, pág. 1).

Con un enfoque tan solo a lo social, se considera que las personas se adaptan a su entorno, dependiendo del lugar en el que viven y tendrán distintos gustos, preferencias, así como también necesidades. Sin embargo, en oportunidades, las personas desean incluirse en otro grupo y es allí donde nuevas preferencias o deseos.

\section{Teoría de la jerarquía de las necesidades}

Autores como García (2014) sostienen que la Teoría de la jerarquía de las necesidades de Maslow, "Forma parte de una teoría psicológica que inquiere acerca de la motivación y las necesidades del ser humano: aquello que nos lleva a actuar tal y como lo hacemos" (pág. 1). Maslow creó esta teoría con el fin de plasmar cómo se deben satisfacer las necesidades, desde las más básicas hasta las más complejas; para ello, las ubicó en una pirámide que consta de cinco niveles.

Siempre ha existido la interrogante de qué motiva el comportamiento humano, "según el psicólogo humanista Abraham Maslow, nuestras acciones están motivadas para satisfacer ciertas necesidades" (Rodríguez, 2018, pág. 1). Esta jerarquía sugiere que las personas primero satisfacen sus necesidades más básicas para que poco a poco vayan satisfaciendo las demás hasta llegar a las más avanzadas. Los niveles establecidos son: Necesidades fisiológicas, necesidades de seguridad, necesidad de relaciones interpersonales, necesidad de reconocimiento y necesidad de autorrealización.

"Maslow en su teoría nos afirma que las personas tienen dos tipos de necesidades: Las deficitarias y las de crecimiento. Estas necesidades están organizadas de for- ma jerárquica, es decir que las primeras se han de satisfacer antes que el resto" (Whaibe, 2013, pág. 10).

Teoría del consumidor

Autores como Vega (2010), señalan que "La teoría del consumidor, trata básicamente de buscar entender y explicar cómo es la asignación que hacen los consumidores de la renta a la compra de los diferentes bienes y servicios, que maximicen su bienestar" (pág. 1). Un consumidor buscará el producto o servicio que le proporcione mayor utilidad y dependiendo de sus ingresos lo adquirirá para poder satisfacer su necesidad.

Esta teoría fue "impulsada por autores como Menger, Jevons y Walras o Alfred Marshall, es de vital importancia en la economía y gestión empresarial. A partir de ella, podemos observar cómo se produce el comportamiento del consumidor" (Gestión estratégica, 2018, pág. 1), refiriéndose a saber con qué bases el consumidor adquiere los productos que podrían satisfacerlo pero siempre se toma en cuenta que tratar de alcanzar su bienestar el presupuesto disponible es de suma importancia.

La publicidad es un medio que las empresas contratan con el fin de informar a los consumidores, ya sea de su existencia, promociones o en sí de sus productos (Thompson, 2017). La publicidad tiene varios espacios en los que puede ser mostrada ya sean en calles, edificios, internet, entre otros; las empresas deben buscar el que mejor se adapte a sus objetivos.

\section{Planteamiento del problema.}

A lo largo de los años varias teorías se han enfocado en el estudio de los diferentes tipos de publicidad en los consumidores, cada una de ellas enfocándose de distinta manera, así como también en diferentes segmentos de mercado. La presente investigación tiene como fin analizar la influencia de la 
publicidad visualizada en buses de transporte público, sobre el comportamiento de los consumidores del transporte público del cantón Rumiñahui, en el período marzo-julio del 2019, considerando dos variables: publicidad visualizada en los transportes públicos y el comportamiento del consumidor.

La variable independiente es la publicidad; en este caso, la visualizada por los consumidores en el transporte público en la localidad objeto de estudio y la variable dependiente es el comportamiento del consumidor, que según (Vega, 2010) se trata sobre cómo los distintos tipos de consumidores adquieren sus productos tomando en cuenta que dicha adquisición dependerá de su nivel económico es decir sus ingresos. Cada consumidor dependiendo de sus necesidades buscará la manera de satisfacerlas (Ver Figura 1)

Figura 1. Modelo de investigación

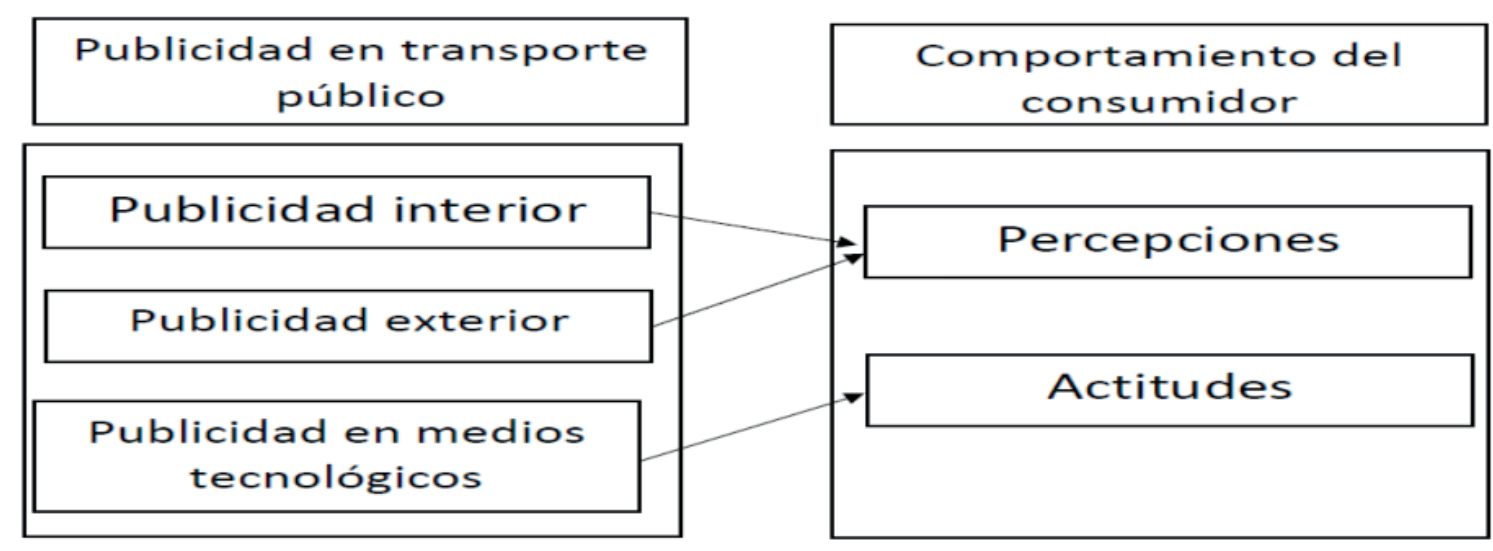

\section{Objetivo}

Identificar si la publicidad en medios de transporte del Cantón Rumiñahui, influye en el comportamiento del consumidor, mediante un estudio metodológico para conocer un nuevo medio de publicidad.

\section{Hipótesis de la investigación}

La publicidad en medios de transporte de Rumiñahui influye en el comportamiento del consumidor.

\section{Método}

Para el presente estudio se seleccionó una metodología desarrollada en dos fases: la primera es la construcción de la perspectiva teórica mediante la revisión de la literatura sobre el tema objeto de estudio. Esta primera fase es elemental para la creación del constructo cognitivo sobre el cual se fundamenta el presente estudio. Esta fundamentación teórica permite acercarse al objetivo de la investigación que consiste en determinar la influencia de la publicidad visualizada en los transportes públicos sobre el comportamiento del consumidor de Rumiñahui, considerando las dos variables: publicidad presentada en medios de transporte, y el comportamiento del consumidor, determinada por los comportamientos y actitudes relativos a las decisiones de consumo de los productos presentados. La segunda fase contempla un trabajo de campo, para ello se recolectará la información a través de un instrumento aplicado a 383 usuarios del transporte público 
(buses) de la localidad seleccionada y en el periodo escogido para tales efectos.

\section{Resultados}

\section{Validez del contenido}

La validez de contenido se realizó a través de la consulta a 8 expertos en el área de mar- keting y de la publicidad, así como a expertos en el área de transporte público urbano como directivos de cooperativas de buses que circulan dentro del perímetro del cantón Rumiñahui, para obtener su criterio y experiencia de la publicidad implementada en las diferentes unidades de transporte de sus cooperativas. Se presenta el siguiente nivel de detalle:

Tabla 1. Detalle de expertos que validaron el contenido del instrumento de recolección de datos

\begin{tabular}{|l|l|l|l|}
\hline $\begin{array}{c}\text { No. } \\
\text { Experto }\end{array}$ & \multicolumn{1}{|c|}{ Género } & \multicolumn{1}{c|}{ Ocupación } & \multicolumn{1}{c|}{$\begin{array}{c}\text { Nivel de } \\
\text { estudios }\end{array}$} \\
\hline 1 & Femenino & Socio directivo de cooperativa Libertadores & Licenciatura \\
\hline 2 & Femenino & Docente Marketing Digital & Ingeniería \\
\hline 3 & Masculino & Directivo de cooperativa de buses Los Chillos & Ingeniería \\
\hline 4 & Masculino & Colaborador de agencia de publicidad & Ingeniería \\
\hline 5 & Femenino & Directivo de agencia de publicidad & Doctorado \\
\hline 6 & Masculino & Docente en instituto de publicidad & Ingeniería \\
\hline 7 & Masculino & Supervisor de agencia de publicidad & Maestría \\
\hline 8 & Femenino & Docente Marketing y Publicidad & Ingeniería \\
\hline
\end{tabular}

Tabla 2

\begin{tabular}{|c|c|c|}
\hline \multicolumn{3}{|c|}{ Masculino y Femenino } \\
\hline Género & Cantidad & $\%$ \\
\hline Doctor & 1 & $12,50 \%$ \\
\hline Ingeniería & 5 & $62,50 \%$ \\
\hline Maestría & 1 & $12,50 \%$ \\
\hline Licenciatura & 1 & $12,50 \%$ \\
\hline TOTAL & 8 & $100,00 \%$ \\
\hline
\end{tabular}

El grupo de expertos estuvo conformado por el 62,50\% de ingenieros (5); el restante $37,50 \%$ se conformó por 1 Doctor, 1 Licenciado y 1 Magíster.

Cada experto se especializa en áreas diferentes que son de gran aporte para esta investigación: el 37,50\% son docentes del área de marketing y publicidad (3); el 25\% son socios o directivos de alguna cooperativa de buses de transporte público (2), y el restante $37,50 \%$ se distribuye entre personas que laboran en una agencia de publicidad, presen- tándose cargos como 1 Directivo, 1 supervisor y un colaborador de dicha agencia.

\section{Validez de contenido del instrumento}

Para la validez de contenidos se tomó el criterio de los expertos quienes calificaron cada pregunta de cada dimensión del estudio, con valores en las escalas de representatividad, comprensión, interpretación y claridad de cada pregunta en una escala del 1 al 3. A continuación se presenta el significado de cada una: 
Tabla 3. Criterios de calificación

\begin{tabular}{|c|c|c|c|}
\hline Representatividad & Comprensión & Interpretación & Claridad \\
\hline $\begin{array}{l}1=\text { Nada representativo, 2 } \\
\text { Neutro, 3 = Muy representativo }\end{array}$ & $\begin{array}{l}1=\text { Incomprensible, 2 } \\
\text { Neutro, 3 = Se compren- } \\
\text { de claramente }\end{array}$ & $\begin{array}{l}1=\text { Se puede interpre- } \\
\text { tar de varias formas, } 2 \\
=\text { Neutro, 3 = Tiene una } \\
\text { única interpretación }\end{array}$ & $\begin{array}{l}1=\text { Nada claro, 2 = Neu- } \\
\text { tro, 3 = Conciso/directo }\end{array}$ \\
\hline
\end{tabular}

Para ver la validez de cada pregunta se toma el criterio que cada pregunta debe tener la calificación mayor a 75\%, por tal razón como se puede visualizar en la tabla 4 , se eliminan 4 preguntas demográficas y
2 preguntas de las dimensiones, las cuales se presentan en este documento resaltado. Esto por no representar importancia según el criterio de los expertos.

Tabla 4. Calificaciones recolectadas de los expertos

\begin{tabular}{|c|c|c|c|c|c|c|c|}
\hline Items & $\begin{array}{l}\text { Represen- } \\
\text { tatividad }\end{array}$ & $\begin{array}{l}\text { Compren- } \\
\text { sión }\end{array}$ & Interpretación & Claridad & Total & $\begin{array}{l}\text { Máximo por } \\
\text { pregunta }\end{array}$ & $\begin{array}{l}\text { Pondera- } \\
\text { ción }\end{array}$ \\
\hline \multicolumn{8}{|l|}{ Demográficas } \\
\hline $\mathrm{D} 1$ & 10 & 16 & 16 & 17 & 59 & 96 & $61 \%$ \\
\hline $\mathrm{D} 2$ & 11 & 17 & 18 & 18 & 64 & 96 & $67 \%$ \\
\hline D3 & 22 & 20 & 19 & 22 & 83 & 96 & $86 \%$ \\
\hline D4 & 24 & 24 & 24 & 24 & 96 & 96 & $100 \%$ \\
\hline D5 & 13 & 18 & 16 & 17 & 64 & 96 & $67 \%$ \\
\hline D6 & 22 & 22 & 22 & 22 & 88 & 96 & $92 \%$ \\
\hline $\mathrm{D7}$ & 14 & 16 & 16 & 19 & 65 & 96 & $68 \%$ \\
\hline D8 & 20 & 20 & 19 & 20 & 79 & 96 & $82 \%$ \\
\hline \multicolumn{8}{|c|}{ Publicidad interna } \\
\hline PI1 & 21 & 22 & 23 & 18 & 84 & 96 & $88 \%$ \\
\hline $\mathrm{PI} 2$ & 22 & 23 & 22 & 19 & 86 & 96 & $90 \%$ \\
\hline $\mathrm{PI} 3$ & 22 & 22 & 22 & 19 & 85 & 96 & $89 \%$ \\
\hline PI4 & 15 & 11 & 14 & 11 & 51 & 96 & $53 \%$ \\
\hline \multicolumn{8}{|c|}{ Publicidad externa } \\
\hline PE5 & 22 & 19 & 20 & 21 & 82 & 96 & $85 \%$ \\
\hline PE6 & 20 & 18 & 21 & 21 & 80 & 96 & $83 \%$ \\
\hline PE7 & 20 & 18 & 19 & 19 & 76 & 96 & $79 \%$ \\
\hline \multicolumn{8}{|c|}{$\begin{array}{l}\text { Publicidad en me- } \\
\text { dios tecnológicos }\end{array}$} \\
\hline PMT8 & 24 & 22 & 22 & 21 & 89 & 96 & $93 \%$ \\
\hline PMT9 & 23 & 19 & 20 & 22 & 84 & 96 & $88 \%$ \\
\hline \multicolumn{8}{|l|}{ Percepciones } \\
\hline P10 & 12 & 11 & 10 & 10 & 43 & 96 & $45 \%$ \\
\hline P11 & 22 & 21 & 20 & 17 & 80 & 96 & $83 \%$ \\
\hline P13 & 19 & 20 & 19 & 18 & 76 & 96 & $79 \%$ \\
\hline P14 & 22 & 22 & 22 & 19 & 85 & 96 & $89 \%$ \\
\hline \multicolumn{8}{|l|}{ Actitudes } \\
\hline A15 & 21 & 24 & 23 & 24 & 92 & 96 & $96 \%$ \\
\hline A16 & 20 & 21 & 20 & 20 & 81 & 96 & $84 \%$ \\
\hline
\end{tabular}


Como resultado de la validación del instrumento de recolección de datos, este queda finalmente planteado con 13 preguntas referentes a las dimensiones de la publicidad en transporte público y del comportamiento del consumidor. Adicionalmente, se plantean 4 preguntas demográficas que permite categorizar las respuestas de los encuestados por características representativas.

\section{Análisis univariado y bivariado}

La investigación de la hipótesis se plantea por medio de análisis mediante el programa SPSS 22. En primer lugar, se realizó un análisis estadístico univariado de frecuencias, como respaldo para la comprobación de cada hipó- tesis a continuación, se efectuó un análisis bivariado con una agrupación de cada una de las preguntas más representativas probadas con el coeficiente de Spearman; para finalmente probar las hipótesis por medio de tablas cruzadas aplicando el estadístico de prueba de chi cuadrado. Los resultados de este análisis son los siguientes:

\section{Comprobación de la hipótesis 1}

Ho: La publicidad interior de los buses de transporte público de Rumiñahui no influye en la percepción de los usuarios de estos medios de transporte.

\section{Estadístico de prueba}

Tabla 1. Coeficiente de chi cuadrado con SPSS 22

\begin{tabular}{|l|l|l|l|}
\hline & \multicolumn{1}{|c|}{ Valor } & \multicolumn{1}{c|}{ Gl } & Sig. asintótica bilateral \\
\hline Chi-cuadrado de Pearson & 52,914 & 4 &, 000 \\
\hline Razón de verosimilitud & 38,364 & 4 & $\mathbf{0 0 0}$ \\
\hline Asociación lineal por lineal & 22,292 & 1 & $\mathbf{0 0 0}$ \\
\hline N de casos válidos & 383 & & \\
\hline
\end{tabular}

Reglas de decisión Grados de libertad: 4 $\alpha=0,05$

Valor crítico: 9,488

Si X2 > 9,488 se rechaza la hipótesis nula y se acepta la hipótesis alternativa caso contrario si $X 2 \leq 9,488$ se acepta la hipótesis nula y se rechaza la hipótesis alternativa.

\section{Prueba de hipótesis con valor $\mathrm{p}$}

Con un nivel de significancia del valor p: 0,05

Si $p \leq \alpha$ se rechaza la hipótesis nula se acepta la hipótesis alternativa.

Si $p>\alpha$ se acepta la hipótesis nula y se rechaza la hipótesis alternativa.

\section{Conclusión}

Según chi critico se tiene un valor de 9,488 a 0,05 grados de libertad y un chi calculado de 52,914. Se puede concluir que la hipótesis nula se rechaza y por defecto se procede a aceptar la hipótesis alternativa demostrando así, que la publicidad interior de los buses de transporte público de Rumiñahui influye en la percepción de los usuarios de estos medios de transporte público.

\section{Comprobación Hipótesis 2}

Ho: La publicidad exterior de los buses de transporte público de Rumiñahui no influye en la percepción de los usuarios de estos medios de transporte. 


\section{Estadístico de prueba}

Tabla 2. Coeficiente de chi cuadrado con SPSS 22

\begin{tabular}{|l|l|l|l|}
\hline & \multicolumn{1}{|c|}{ Valor } & \multicolumn{1}{c|}{ Gl } & \multicolumn{1}{c|}{ Sig. asintótica bilateral } \\
\hline Chi-cuadrado de Pearson & 86,914 & 4 &, 000 \\
\hline Razón de verosimilitud & 72,994 & 4 &, $\mathbf{0 0 0}$ \\
\hline Asociación lineal por lineal & 59,519 & 1 &, 000 \\
\hline N de casos válidos & 383 & & \\
\hline
\end{tabular}

Reglas de decisión Grados de libertad: 4 $\alpha=0,05$

Valor crítico: 9,488

Si $X_{2}>9,488$ se rechaza la hipótesis nula y se acepta la hipótesis alternativa caso contrario si $X_{2} \leq 9,488$ se acepta la hipótesis nula y se rechaza la hipótesis alternativa.

\section{Prueba de hipótesis con valor $\mathrm{p}$}

Con un nivel de significancia del valor p: 0,05

Si $p \leq \alpha$ se rechaza la hipótesis nula se acepta la hipótesis alternativa.

Si $p>\alpha$ se acepta la hipótesis nula y se rechaza la hipótesis alternativa.

\section{Conclusión}

Según chi critico se tiene un valor de 9,488 a 0,05 grados de libertad y un chi calculado de 86,914 se puede concluir que la hipótesis nula se rechaza y, por defecto, se procede a aceptar la hipótesis alternativa. Esto demuestra que la publicidad exterior de los buses de transporte público de Rumiñahui influye en la percepción de los usuarios de estos medios de transporte público.

\section{Comprobación hipótesis 3}

Ho: La publicidad en medios tecnológicos de los buses de transporte público de Rumiñahui influye en la actitud de los usuarios de estos medios de transporte.

\section{Estadístico de prueba}

Tabla 2. Coeficiente de chi cuadrado con SPSS 22

\begin{tabular}{|l|l|l|l|}
\hline & \multicolumn{1}{|c|}{ Valor } & \multicolumn{1}{|c|}{ Gl } & \multicolumn{1}{c|}{$\begin{array}{c}\text { Sig. asintótica } \\
\text { bilateral }\end{array}$} \\
\hline Chi-cuadrado de Pearson & 104,860 & 4 &, 000 \\
\hline Razón de verosimilitud & 80,123 & 4 &, 000 \\
\hline Asociación lineal por lineal & 69,508 & 1 &, 000 \\
\hline N de casos válidos & 382 & & \\
\hline
\end{tabular}

Reglas de decisión Grados de libertad: 4 $\alpha=0,05$ Valor crítico: 9,488
Si X2 > 9,488 se rechaza la hipótesis nula y se acepta la hipótesis alternativa caso contrario si $X 2 \leq 9,488$ se acepta la hipótesis nula y se rechaza la hipótesis alternativa. 


\section{Prueba de hipótesis con valor $\mathrm{p}$}

Con un nivel de significancia del valor p: 0,05

Si $p \leq \alpha$ se rechaza la hipótesis nula se acepta la hipótesis alternativa.

Si $p>\alpha$ se acepta la hipótesis nula y se rechaza la hipótesis alternativa.

\section{Conclusión}

Según el chi crítico, se tiene un valor de 9,488 a 0,05 grados de libertad y un chi calculado de 104,860 se puede concluir que la hipótesis nula se rechaza y, por defecto, se procede a aceptar la hipótesis alternativa demostrando así que la publicidad en medios tecnológicos de los buses de transporte público de Rumiñahui influye en la actitud de los usuarios de estos medios de transporte.

\section{Conclusión de la investigación}

Los resultados de esta investigación mostraron con claridad que la publicidad implementada en los buses de transporte público influye positivamente en el comportamiento de los consumidores del Cantón Rumiñahui. Este comportamiento positivo de las personas beneficiará directamente a las marcas que deseen publicitar en estos medios de transporte.

Este estudio, además, resulto factible para conocer la percepción de los usuarios que se basa en las necesidades, deseos, valores y experiencias vividas que tienen los usuarios o ciudadanía sobre este tipo de publicidad. De manera especial, se resaltó que la única manera de llegar al consumidor no es la publicidad tradicional (radio, prensa y televisión), especialmente a la población que pasa el día a día transportándose en la ciudad ya sea por trabajo o estudio.

\section{Referencias bibliográficas}

Asenjo, Y. (24 de enero de 2019). Docsity. Obtenido de Teoría de los efectos limitados: https://www.docsity.com/es/teoria-delos-efectos-limitados-1/4420828/

Bardales, A. P. (18 de agosto de 2017). Merkactiva. Obtenido de Teorías del comportamiento del consumidor: http://www.merkactiva. com/blog/teorias-del-comportamientodel-consumidor/

Cantoral, A. (2015). UDLAP. Obtenido de Teoría de la Aguja Hipodérmica: http://web.udlap. mx/co21502/primeros-estudio/

Consumer Behavior. (2014). Obtenido de Modelo psicológico social de Veblen: https:// sites.google.com/site/consumerbehavior2dnm3/modelo-psicologico-socialde-veblen

Flores, E. (2013). Evaluación de la publicidad BTL en asientos de buses interprovinciales para la creación de una empresa. Repositorio Universidad de Guayaquil.

Gálvez, J. Á. (2012). Universidad Complutense. Obtenido de Modelos teóricos sobre los efectos de los medios de comunicación de masas: https://eprints.ucm.es/45089/1/ doc\%20trab\%202.pdf

García, J. (2014). Psicología y mente. Obtenido de Pirámide de Maslow: la jerarquía de las necesidades humanas: https://psicologiaymente. com/psicologia/piramide-de-maslow

Gestión estratégica. (2 de Febrero de 2018). Escuela Europea de Management. Obtenido de Teoría del comportamiento del consumidor: conceptos básicos: http://www. escuelamanagement.eu/gestion-estrategica/teoria-del-comportamiento-del-consumidor-conceptos-basicos

Martin, M., y María, A. (2007). Nuevas tendencias en la publicidad en el siglo XXI. Comunicacion social ediciones y publicaciones(1 edición).

Rodríguez, E. (2 de junio de 2018). La mente es maravillosa. Obtenido de Los cinco niveles de la jerarquía de necesidades de Maslow: https://lamenteesmaravillosa.com/loscinco-niveles-de-la-jerarquia-de-necesidades-de-maslow/ 
Rodríguez, J. (2016). La publicidad como herramienta de las distintas modalidades de comunicación persuasiva. Global Media Journal Edición Iberoamericana , 17-25.

Teoriascom. (03 de julio de 2015). Teoriascom. Obtenido de Teoría de los efectos limitados: http://teoriascom.blogspot. es/1435895286/teoria-de-los-efectoslimitados/

Thompson, I. (2017). Definición de publicidad. Marketing, 50-62.

Vega, N. A. (29 de Octubre de 2010). Obtenido de Teorías del consumidor: https://www.
monografias.com/docs/Teoria-del-consumidor-F36TPKK699CP

Villarejo, A. (2018). Comortamiento del consumidor ante los precios promocionales. Conocimiento, innovación y emprendedores, $50-62$

Whaibe, E. (19 de Septiembre de 2013). Instituto Tecnológico de Apizaco. Obtenido de https://www.academia.edu/6109474/ TEORIAS_DEL_COMPORTAMIENTO_DEL_ CONSUMIDOR_COMPLETO?auto=download 